\title{
Affective Level Design for a Role-Playing Videogame Evaluated by a Brain-Computer Interface and Machine Learning Methods
}

\author{
Fabrizio Balducci · Costantino Grana · Rita Cucchiara
}

Received: date / Accepted: date

\begin{abstract}
Game science has become a research field which attracts industry attention due to a worldwide rich sell-market. In order to understand the player experience, concepts like Flow or Boredom mental states require formalization and empirical investigation, taking advantage of the objective data that psychophysiological methods like electroencephalography (EEG) can provide. This work studies the affective ludology and shows two different game levels for Neverwinter Nights 2 developed with the aim to manipulate emotions; two sets of affective design guidelines are presented, with a rigorous formalization that considers the characteristics of Role-Playing genre and its specific gameplay. An empirical investigation with a brain-computer interface headset has been conducted: by extracting numerical data features, machine learning techniques classify the different activities of the gaming sessions (task and events) to verify if their design differentiation coincides with the affective one. The observed results, also supported by subjective questionnaires data, confirm the goodness of the proposed guidelines suggesting that this evaluation methodology could be extended to other evaluation tasks.
\end{abstract}

Keywords Affective design · Role-Playing games · Brain-Computer Interface

F. Balducci, C. Grana, R. Cucchiara

Dipartimento di Ingegneria "Enzo Ferrari"

Università degli Studi di Modena e Reggio Emilia

Via Vivarelli 10, Modena (MO) 41125, Italy

Tel.: +390592056265

Fax: +390592056129

E-mail: fabrizio.balducci@unimore.it,

costantino.grana@unimore.it,rita.cucchiara@unimore.it

\section{Introduction}

The concept of playing is strictly linked to our lives, one of the first activities that a human experiences by discovering his surrounding world: a successful method to learn rules or how to safely execute a task is in fact "gamify", i.e. to see them like a gaming activity from which gaining experience for future goals [1]; this is the basis of gamification and serious gaming theories which try to introduce playful aspects, often borrowed by videogame creators, in all daily interactive activities.

Lindley [2] provides a commonly accepted definition of game as "a goal-directed and competitive activity conducted within a framework of agreed rules". Given this definition, it is often said that "to play" involves learning the internal rules of a game, i.e. the set of mechanics that characterize its interactive dynamics and denote each game genre (known as "gameplay").

Frasca [3], referring to Csíkszentmihályi's studies [4], defines the modern concept of ludology as the "science of the game" which uses research methods and theories from a wide range of scientific communities (such as Human-Computer Interaction and psychophysiology) with the aim to improve the methodologies to study both players and (video)games, understanding the design of an optimal player experience.

In over 30 years videogame platforms evolved, moving from large old cabinets to smart-watches that easily permit to play in mobility, allowing learning [5], large data exploration [6] or archaeological visits [7]. The academic world also considers videogaming as a multidisciplinary research [8] and teaching field [9].

Nowadays the videogame represents a medium that requests high skilled workers [10] and budgets that can rival with cinema, music, comics and literature industry. Videogames production is a large business [11] esti- 
mated in $93 \$$ billion in 2013 [12]. The development cost of Call of Duty Modern Warfare 2 was between $40 \$$ million - 50\$ million, with a marketing and release budget of $\$ 200$ million [13]. Other aspects that highlight the market widening are the intersection between toys and games [14], the phenomenon of in-game advertising [15] and the importance of press and user critics [16].

The costs for a triple $A$ title oblige editors to accept fewer risks, looking for a formula that will ensure great games for everyone, omitting that each person is different due to factors like age, education, country and personal history. While Paavilainen [17] and Korhonen et al. [18] propose and review many evaluation heuristics, it becomes crucial to define and manipulate complex concepts like Fun with innovative methods that consider player's feelings and preferences by retrieving objective and scientifically evaluable data.

This paper describes how to apply affective level design and the manipulation of emotions in digital Role-Playing Games by designing activities in a game level (also known as "scenario"); moreover a novel evaluation methodology is proposed, to extract and analyze objective data from affective states and use them to uniquely connote the activities and the game level areas formally developed.

Section 2 presents experimental studies about gaming while Section 3 introduces terms commonly used in videogames design; a brief introduction to emotion analysis is provided in Section 4. Section 5 proposes formal design guidelines and Section 6 illustrates their practical application into a commercial RPG videogame. Section 7 briefly illustrates the technical tools used while Section 8 describes the experimental setup. Results from experimentation and their evaluation are in Section 9 while Section 10 focuses on questionnaires data. Finally, conclusions are drawn in Section 11, along with suggestions for future work.

\section{Related work}

The manifestation of mental states in a person can be measured: studies from Mandryk et al. [19] have successfully demonstrated how psychophysiological techniques such as electroencephalography (EEG) evidence human emotions and cognitive activity during gaming. Affective computing, as a field of study, was captured by Rosalind Picard [20] while Nacke [21] introduces the concept of affective ludology referring to the investigations of affective player-game interaction to understand emotional and cognitive experiences: it must inquire about cognition, emotions, and goal-oriented behavior from a scientific perspective and establish rigor- ous methodologies (e.g. psychological player testing or physiological response analysis of players).

Noah et al. [22] measure brain activity with magnetic resonance imaging (MRI) using a clone of Dance Dance Revolution videogame to study how different sensory inputs influence the motor output, while Groenegress et al. [23] introduce a system for realtime physiological analysis and metaphorical visualization within a virtual environment, considering heart rate, respiration and galvanic skin responses. Rawn and Brodbeck [24] use questionnaires and Doom 3 to inquire about violence and aggressive interactions while Jacopin [25] analyzes internal data from F.E.A.R., KillZone 3 and Transformers 3 to develop intelligent NPC; in Gilleade et al. [26] the affective state is used to manipulate the game session.

A Brain-Computer Interface is a system that measures brain electrical activity allowing to retrieve information about feelings and emotions. In the field of computer science, the BCI has been used with various purposes like measure mnemonic and cognitive efforts (Grimes et al. [27]) while Vachiratamporn et al. [28] measure player experience with EEG and heartbeat signals, using a survival horror videogame as experimental environment. Lotte [29] considers BCI as an additional control channel to interact with virtual environments, but its results underline some limits according to which $20 \%$ of players cannot use it as a gaming device; adaptive virtual environment and emotion assessment methods are also in [30] while Coulton et al. [31] successfully use EEG headset with mobile games.

Burke et al. [32] introduce game design guidelines for stroke rehabilitation using serious games and Kang et al. [33] create a 3D sensory gate-ball game system to improve both physical and mental health of the aged people. Nacke et al. [34,21] evaluate game levels for the first-person shooter Half-Life using a BCI while [35] consider to evaluate videogames dealing with game activities like events (an automatically activated accident) and tasks (a voluntary performed act).

Another way to employ an EEG headset is like a real-time input device: Vourvopoulos et al. [36] propose a reconstruction of the ancient Rome that can be explored using brainwaves and also Liarokapis et al. [37] create a Lego NTX robot remotely controlled by them; in both studies there is a comparison between the headsets produced by Emotiv and Neurosky. Finally Schwarz et al. [38] introduce a Pong clone controlled by two Emotiv headset together and Chumerin et al. [39] propose an application where an avatar navigates The Maze guided by brainwaves. 


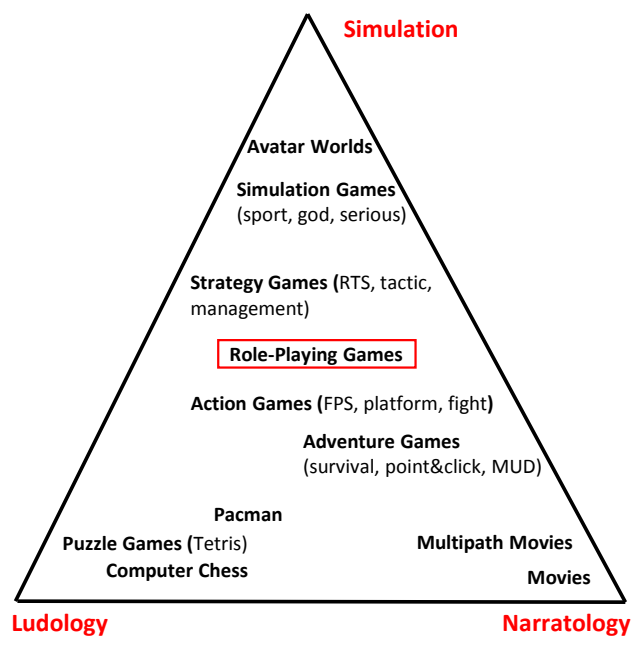

Fig. 1 The triangular scheme that illustrates the polarity of game genres, according to three metrics from which the gameplay is characterized.

\section{Gameplay and Genres}

The design of a videogame depends on the gameplay and the genre: the former defines how narrative and ludic elements guarantee that players don't quit the game [40], while the latter exposes design solutions and constant features coming from previous products widely accepted by the sell-market; usually a gamer refers to a videogame citing its genre or by a direct comparison stating that game $X$ is like $Y$ (already known) [41]. Defining a gameplay involves the tacit knowledge of designers whose target is to maximize the player experience, i.e. the set of feelings and opinions that come from an activity which has to capture player's senses.

The term "genre" is used to classify and categorize entities by highlighting their common and uncommon features, as it happens for books and movies. In the videogame context it refers to which gameplay a gamer wants to satisfy his player experience. Machado et al. [42] develop a player modeling taxonomy with several game platforms that can be used by researchers, Lewis et al. [43] propose a taxonomy for game bugs while Pinelle et al. [44] consider how genres concern usability and design issues.

Compiling a taxonomy with a clear genres division involves much subjectivity due to the extreme variety of gameplay: the works of Berens and Howard [45] and Carlá [46] suggest classifications based on historical period and technology platforms. Khaleghi and Lugmayr [47], and Scavarelli and Arya [12], introduce respectively surveys and a game ethics framework (tested on Fable and Super Mario Bros.) to classify according to genre, technologies and gameplay features.

Lindley [2] defines a triangular scheme (a version of which is in Fig. 1) where each vertex denotes the polarization of genres towards the metrics of Simulation, Ludology and Narratology. Privileging Simulation implies the reconstruction of physical laws and real world constraints (or particular aspects of them), denoting a slow gameplay based on parameters tuning and high longevity; conversely Ludology denotes immediate frantic gameplay with progressive difficulty, in fact this games belong to the arcade genre which is characterized by few options but low longevity.

Langer et al. [48] propose suspenseful design to inquire narrative and storytelling, while Frasca [49] studies the relationships between ludology and narrative considering it as an integration for other metrics, in fact it offers ways to enhance involvement similarly to non-interactive media. Calleja [50] studies the narrative involvement, introducing a model to understand the player experiential narrative, while Zagalo and Torres [51] present an emotion module from an authoring tool of interactive storytelling.

It is noteworthy that in the scheme in Fig. 1, Role-Playing genre is positioned exactly in the center, emphasizing the balance of the metrics: in Section 5 and 6 a further analysis of these factors will bring to the formalization of general design guidelines.

\section{Emotions and Affective States}

The electric potential generated by a single brain neuron is too weak to be detected by an electroencephalogram, and in fact it is the synchronized activity of millions of neurons having the same spatial orientation that is considered. For their characteristics, the pyramidal neurons on the cerebral cortex are considered the largest emitters of EEG signals producing different brainwaves characterized by their frequency [52]:

- Gamma Waves have the highest frequency range (30-80 Hz), involved in higher cognitive functioning like memory and information processing; states of anxiety and stress presents high levels of this.

- Beta Waves known as "high frequency-low amplitude" waves (13-30 Hz) involves conscious thought, logical and critical thinking and socialization; their activity increases with stimulants, like caffeine.

- Alpha Waves associated to calm and meditation with a regular and synchronized configuration (8-13 $\mathrm{Hz}$ ); they are also called Berger waves in memory of the inventor of the EEG in 1929. 


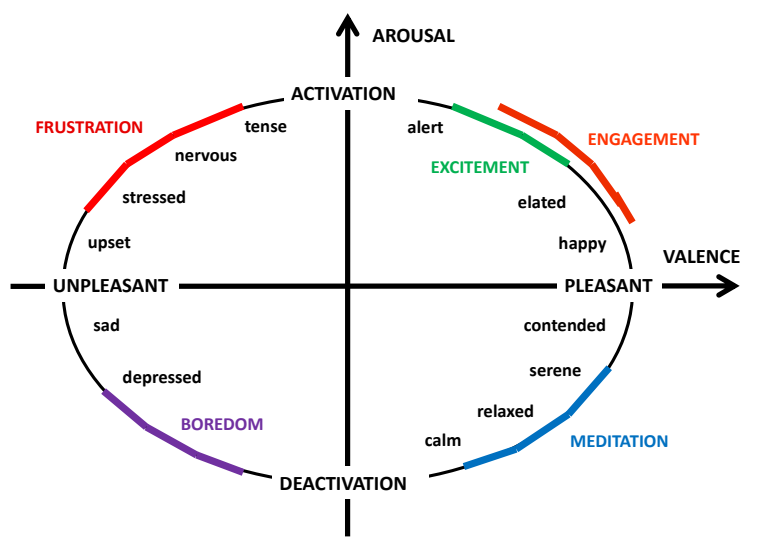

Fig. 2 A circumplex model of affect interpretation: Excitement is pleasant and activated so it is located in the upperright quadrant like Engagement. Meditation, pleasant but not activated, is located in the bottom-right quadrant. Frustration and Boredom are unpleasant, but the first is activated and so it is located in the upper-left quadrant while the second is in the bottom-left.

- Theta Waves involved in daydreaming and REM sleep phase with a low frequency range $(3.5-8 \mathrm{~Hz})$; they are connected to deep and raw emotions, intuition and creativity with streams of consciousness near an hypnotic state.

- Delta Waves the slowest recorded brainwaves (under $3.5 \mathrm{~Hz}$ ) associated with deepest levels of sleep; an abnormal activity usually denotes brain injuries and learning problems.

An useful scheme to evaluate basic emotions is the Circumplex model of affect by Russell et al. [53,54] (an interpretation of which is in Fig. 2): it consists of a twodimensional spatial model with a set of human emotions defined on a circumference, related to the bipolar metrics of Valence (X axis) and Arousal ( $\mathrm{Y}$ axis). Valence is an indicator which describes if an emotion is pleasant or unpleasant, while Arousal denotes the intensity (activation or deactivation), i.e. reactivity to stimuli that influence its detection.

Andreassi [55] defines psychophysiology as the science which inquires relations between the psychological manipulation and the resulting physiological effects, linking physiological measures (EEG) with abstract psychological constructs like Attention and Fun; the work of Craveirinha and Roque [56] offers a theoretical overview of the nature of play activities, and studies how certain ludic elements are in relationship with the emotional spectrum.

In Jennett et al. [57] Flow mood clearly overlaps with Immersion in the sense of distorting time: introducing the concept of cognitive absorption, Immersion is proposed as a gradual experience which involves the re- moval of the external environment (spatial, audio-visual and temporal) and appears evidently a precursor for the Flow. Multi-sensory virtual environments to increase Immersion are also in Chalmers et al. [58].

The terms "affect" and "emotion" are often used interchangeably referring to a short-time emotional peak while, conversely, a "mood" or "affective state" denotes a continuous lasting emotional trend which may involve more emotions influencing the global reactions [49]. This work will focus on the manipulation of emotions and on the definition of Flow and Boredom states, considering the five emotion detected by the Emotiv EPOC EEG headset (Excitement, Engagement, Frustration, Meditation and Long-Term Excitement).

Engagement is associated to participation and attention and its increase denotes challenging tasks and pleasure to discover new aspects of the game; commonly it is used as an indicator of Fun. If the aim is to induce a Flow state, most of the game tasks must increase this emotion, while the contrary must be true for the Boredom induction, because it is a deactivated emotion interpretable as the opposite of Engagement.

Excitement is a positive indicator characterized by muscle tension and increased sweating and heartbeat: since evoked by short-time emotional peaks, it is better to consider it linked to Long-Term Excitement i.e. a global value which expresses how stable over time Excitement is. The manipulation of this emotions must work in the same way as seen for Engagement.

Inducing and analyzing Frustration and Meditation is difficult due to their high subjectivity and the negative valence. A meditative activity [59] can take a long time and a lot of training to be correctly performed by a motivated subject, also inducing frustration or boredom in the first attempts.

Frustration might influence other emotions, in fact different players might perceive the same amount of it as a challenge or as a reason to quit the game: if the cause is internal (laziness, lack of confidence) it can be a motivating force but, if caused by external factors perceived to be outside individual control (i.e. task too hard) it can lead to powerlessness and eventually anger (Dollard et al. [60]).

\section{Design for Affective Ludology}

A representation of game levels is described in Park and Park [61] in terms of event/state/action graphs in order to minimize design anomalies while Vanhatupa [62] presents guidelines for RPG games. Thong [63] investigates effectiveness of role-playing videogames as a learning experience emphasizing storyline and characters; 
Horsfall and Oikonomou [64] shows that gamers prefer strong storylines and character development while also Tychsen et al. [65] find that discovery \& immersion are the best motivational features to play them. When considering role-playing gameplay features, we will focus on the aspects shown in Lankoski [66]:

1. strong story plot with moral choices

2. progression of skills and abilities

3. presence of allies in a group (the "party")

4. interaction based on dialogues (question driven)

5. a world to explore (dungeons)

The RPG gameplay is less immediate than other genres because it is based on statistics, skills and object inventory, with a great importance given to environmental exploration; according to Brown and Cairns [67] there are three participation phases (Engagement, Engrossment, Total Immersion) in which the concepts of Immersion and Flow result to be very close.

About the design for Immersion, we consider Calleja [68] who states that player involvement is a prerequisite for it: a captivating story plot, for example, will not only influence the sense of narrative involvement but also the affective involvement, impacting on the quests and goals presented to the gamer (ludic involvement). Nacke et al. [34] propose formal design guidelines that separate Immersion and Flow by dividing the concept of environment from that of combat (which is the focus of FPS gameplay) while Wilcox [69] considers Immersion linked to the realism (of graphic and gameplay) reached by modern videogames .

Keeping these facts in mind, for RPG we consider Immersion as a necessary condition to reach the Flow state: the importance of the visual assets becomes evident for us, in fact it can be considered a "theatrical stage" where characters perform activities. In order to stress these concepts, the first game level created for Neverwinter Nights 2 will focus on the Boredom state while the other will stress the Flow one.

\subsection{Boredom game level}

Fisher [70] defines the boredom like an unpleasant affective state with lack of concentration and difficulty during the execution of a task; Csíkszentmihályi [71] furthermore denotes it like a state in which player's skills are greater than required (Fig. 3-a).

This game level must be characterized by linearity and repetitiveness with poor challenge, minimal plotstory and weak visual assets; dialogues will be short and plain while allies will result unnecessary and weak.

Considering the previous list, the formal design guidelines are:

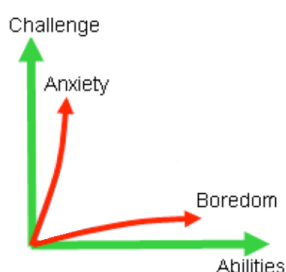

(a)

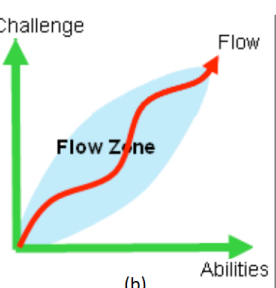

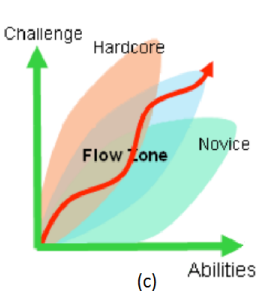

Fig. 3 The Flow state: (a) absence of Flow for unskilled or over-skilled players; (b) Flow with challenge and abilities constant balancing; (c) different Flow zones for different players.

1. Given $n$ sets of different assets like textures $X_{1}$, $3 \mathrm{D}$ models $X_{2}$, enemy types $X_{3}$, sounds $X_{4}$, then a game level $L=\left\{\widetilde{X}_{1}, \widetilde{X}_{2}, \ldots, \widetilde{X}_{n}\right\}$, with $\widetilde{X}_{k} \subset X_{k}$ becomes less interesting if it contains few assets, so that $\forall \widetilde{X}_{k} \in L,\left|\widetilde{X}_{k}\right| \ll\left|X_{k}\right|$; the same should be for all other structural and visual assets like animations, visual effects, items, weapons.

2. Assuming a spatial progression point $p$ to indicate game level progression and given a set $E$ of $n$ types of enemy $e$ with constant strength smaller than player strength, then the player challenge function $f_{c h}(e, p)$ for an encounter with enemy type $e$ at progression point $p$ should remain constant $\forall e \in$ $E, \forall p$.

3. For each progression point $p$ the player should be constantly rewarded with $n$ items that refresh player health value $i_{h}$ and its ammunition value $i_{a}$ so that the full player ammunition supply value $A$ and the full player health value $H$ are steadily reached.

4. No real main goals $G$ are given so that the victory condition doesn't allow any reward to the player.

5. Considering $n$ characters $a$ featuring skills of a specific class $c \in C=\{$ warrior, wizard, thief, animal, unskilled $\}$ and given that $a_{c}$ can join the player's ally party $A$, then $L$ becomes less interesting if $|A|=$ $n$ and $L$ contains much less elements of $A$ so that $L=\left\{a_{1}, \ldots, a_{m}\right\} \subset A, m \ll n \wedge \exists a_{c}, c=$ unskilled.

6. Given $n$ generic dialogues $d \in D=\left\{d_{1}, d_{2}, \ldots, d_{n}\right\}$ that a player can have with the NPCs (Non-Player Characters) of the game level $L$, then $L$ becomes less interesting if contains much less elements of $D$ so that $L=\left\{d_{k}, \ldots, d_{m}\right\} \subset D m \ll n$; given also $n$ questions $q \in Q=\left\{q_{1}, q_{2}, \ldots, q_{n}\right\}$ that the player can choose during an articulated dialog $d_{a} \in D$, then $L$ becomes less interesting if the dialogues contain much less elements of $Q$ so that for the most part of $D$ is $d_{a}=\left\{q_{1}, \ldots, q_{m}\right\} \subset Q, m \ll n$.

\subsection{Flow game level}

The Flow state is characterized by constant balance between challenge and skills (Fig. 3-b): if challenge be- 
comes higher then the activity will result overwhelming generating anxiety while the contrary will provoke boredom: to remain in a Flow state, the player must continue to learn new skills (Broin [72]). Warpefelt [73] propose believable NPCs for player's attention while Burelli [74] investigates how camera behavior impacts on the player experience.

The notion of Flow is closely related to that of Fun but it doesn't coincide with it: Nakamura et al. [75] observed chess and sport players, noting that their enjoyment derives by the mere fact to accomplish their activity independently by other rewards.

Juul [76] studies the factor of difficulty perceived by players, arguing that nowadays videogames have become more easy; Zagal and Altizer [77] analyze mechanisms for character progression as a fundamental element of an RPG, while James et al. [78] faces the concept of reward in games, introducing a differentiation between personal/material/competitive.

A factor which influences the Flow zone is the gamer expertise which may require gameplay variations: in order to design a game for broader audiences, the ingame experience can not be linear and static but instead it needs to offer a wide coverage of potential experiences to fit different players (i.e. the expansion of the Flow zone Fig. 3-c). Due to the previous considerations and with a game design perspective, there are three core elements needed to evoke the Flow (Chen [79]):

- the game is intrinsically rewarding and the player desires to play it

- the game offers the right amount of challenge to match player's abilities

- player feels a sense of personal control on the activities and on the user interface

In this game level, challenges involve complex dialogues and multiple goals; at least one ally helps player to accomplish profitable activities while the level structure includes narrative elements that encourage the environmental exploration.

The specific formal design guidelines are:

1. Given a set of indoor level parts $I$ and a set of outdoor level parts $O$, the game level $L$ should be a set union of outdoor and indoor level parts $L=\{I, O\}$.

2. Given $n$ sets of different assets like textures $X_{1}$, visual effects $X_{2}$, animations $X_{3}$, sounds $X_{4}$, then $L$ becomes more atmospheric and fosters imagination if $L=\left\{X_{1}, X_{2}, \ldots, X_{n}\right\}$; the same should be for all other structural and visual assets like enemy types, items, 3D models, weapons.

3. Assuming a spatial progression point $p$ to indicate a game level progression and given a set $E$ of $n$ type of enemies $e$, then the player challenge function $f_{c h}(e, p)$ for an encounter with an enemy $e$ at progression point $p$ has to progressively increase $\forall e \in E$.

4. For a spatial progression point $p$, after a set of progression interval $p_{k}$ in a game level $L=$ $\left\{p_{1}, p_{2}, \ldots, p_{n}\right\}$, a reward type $r_{k}$ from a set $R=\{$ ammunition, health pack, experience points, money, spell, magic item, weapon, armor $\}$ should be given to the player.

5. There's at least one main goal $G_{k}$ so that the victory condition $V=G_{1} \wedge G_{2} \wedge, \ldots, \wedge G_{n}=$ true; the achievement of $V$ must lead to one or more reward $r_{k}$ from a set of $n$ rewards $R=\left\{r_{1}, r_{2}, \ldots, r_{n}\right\}$ in order to gratify the player's effort.

6. Considering $n$ characters $a$ featuring skills of a specific class $c \in C=\{$ warrior, wizard, thief, animal $\}$ and given that $a_{c}$ can join the player's ally party $A$, then $L$ becomes more interesting if $L \subseteq A$.

7. Given $n$ meaningful dialogues $d \in D=$ $\left\{d_{1}, d_{2}, \ldots, d_{n}\right\}$ that a player can have with the NPCs of the game level $L$, has to be $L \subseteq D$; given also $n$ questions $q \in Q=\left\{q_{1}, q_{2}, \ldots, q_{n}\right\}$ that the player can choose during an articulated dialog $d_{a} \in D$, assuming that $\forall q_{k} \in Q, a n_{k}$ is an answer, then the game level $L$ becomes more interesting if for the most part of $D$ is $d_{a} \subseteq Q \wedge a n_{k}$ is a narrative or ludic element for reaching a victory condition $V$, achieve a reward $r_{k}$ or obtain meaningful information.

\section{Develop for Affective Ludology}

This section, referring to the formalities of the previous one, shows the actual implementation of the two game levels (consisting of smaller pieces called "areas"); the maps in Fig. 4 and Fig. 5 shows player's paths (also optional ones) and provide symbols to indicate game tasks and activities.

\subsection{Boredom game level}

Three areas have been designed by editing the 2311_tunnels original game area that has linear guided paths and poor ambient structures (Fig. 6).

This level consists of only one indoor cave environment, characterized by cold texture colors to emphasize ambient chilliness and repetitiveness; there are no visual effects, no background music or battle sounds and every NPC has the same 3D model.

The story plot offers a very simple task to accomplish without any gratification and the player can't upgrade its character due to the lack of experience points; 


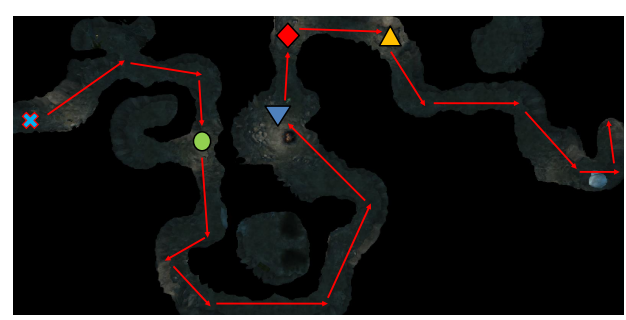

(a) Area 1: beginning of caves

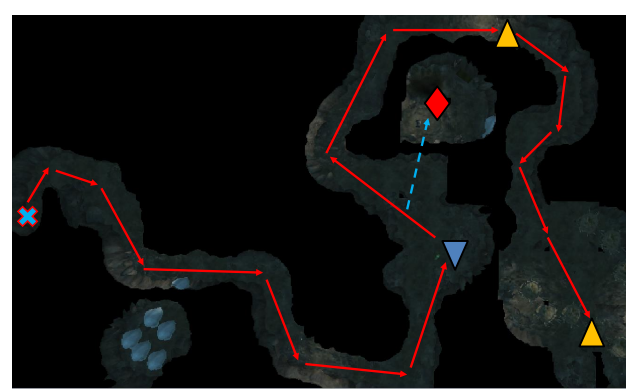

(c) Area 3: the reverse of the previous two

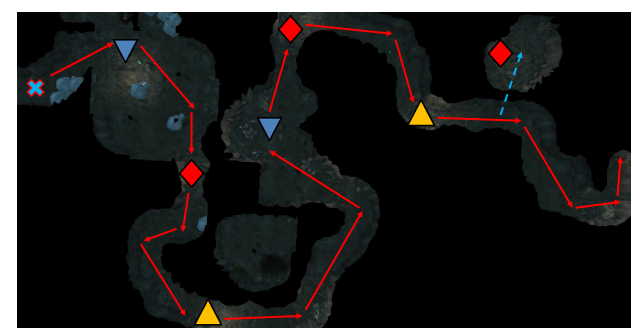

(b) Area 2: the same of the previous one

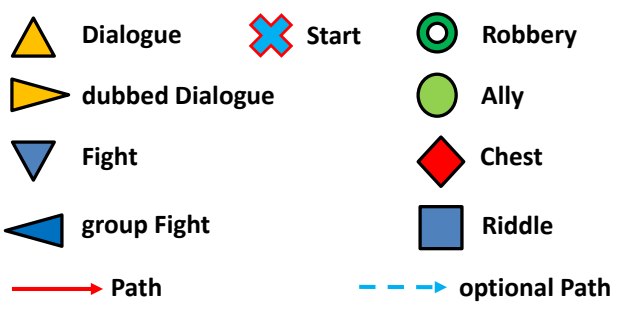

(d) Symbols and meaning

Fig. 4 Boredom game level areas and symbols description.

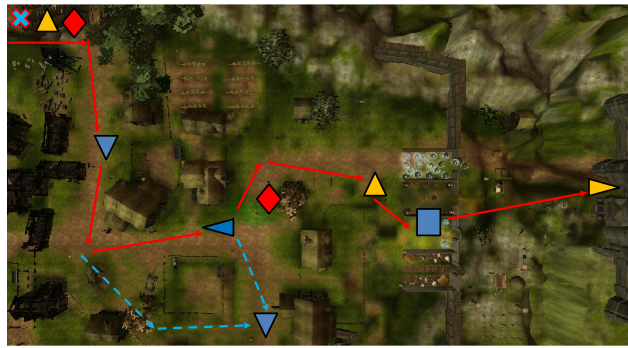

(a) Area 1: countryside

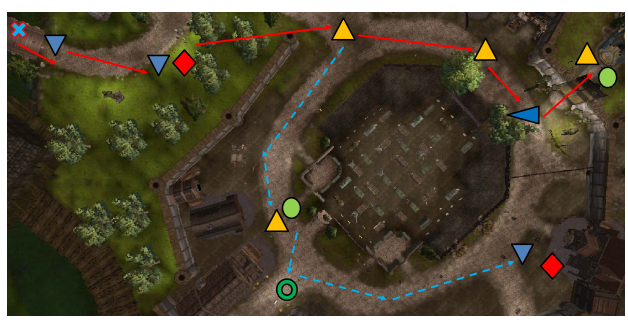

(c) Area 3: haunted village

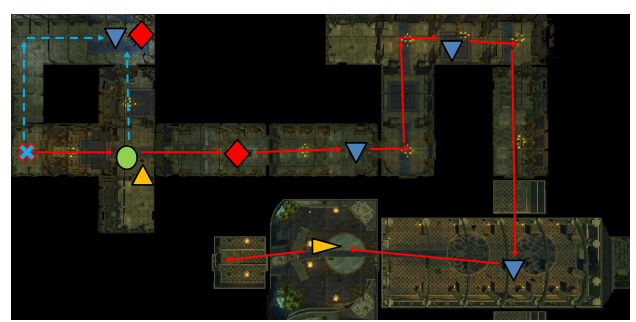

(b) Area 2: indoor castle

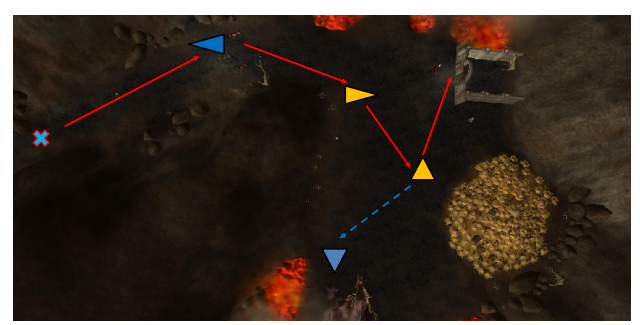

(d) Area 4: volcanic cave

Fig. 5 Flow game level map constituted by four sequential areas.

challenge is minimal with constant difficulty: enemies lack resistance and combat skills and they don't attack in groups (Fig. 6-a). Only two weapons are available without magical skills and player's health and munitions are always kept at the maximum value: every dead enemy releases an health potion and every opened chest contains munitions and weapons.

The unskilled non-interactive ally is unnecessary and tedious, in fact he only follows the main character around the screen using a scripted path-find algorithm; just some NPCs interact with the player using only textual dialogues (no animations or camera changes, Fig. 6-b) that are unbranched and useless, filled by outof-context sentences and with few answers to choose from.

The first Area is linear and empty, introduces the ally, and the only dialogue creates false expectations about the prosecution of the adventure. The second one follows the same path of the previous one, increasing the repetitiveness and lowering the player's expectations. 


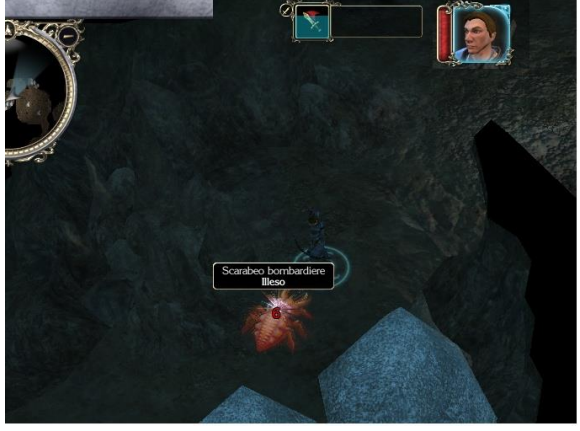

(a) The "single fight" task

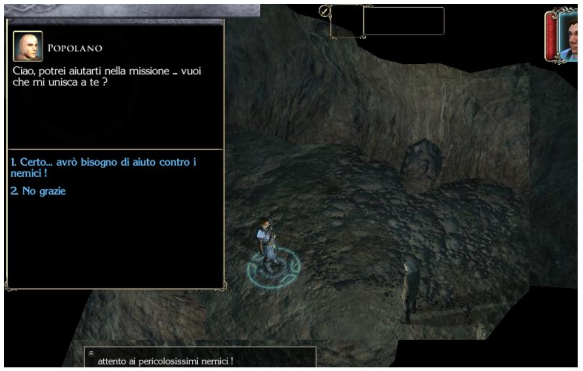

(b) The "dialogue" task only in textual mode

Fig. 6 Tasks in the Boredom game level.

The final area forces the player to trace back along the same path as before leaving him disorientated and free to roam without any real conclusion.

\subsection{Flow game level}

Based on the formal guidelines, the level has been designed by linking and editing the 3010_highcliff, 3000_castle_never, 3063_merchant and 3032_th_canyon original game areas (Fig. 7).

Environmental structures are diversified by indoor and outdoor areas, each of which rich of 3D models, items, visual effects (lights, animated water and trees), weapons, musics and sounds, alternation between day and night; optional paths and tasks introduce variations usually appreciated from completist gamers.

A new articulated and progressive story plot offers well-defined and satisfactory goals (rescue, enrollment, battles), while challenges steadily increase due to the variety of enemy types (with better weapons and skills): previous encountered enemies become harder to defeat and can perform group attacks and ambushes (Fig. 7-a) using spells and magical abilities. Rewards are balanced with skills and challenge: only few enemies (harder to defeat) release health potions while the chests with magic objects, ammo and weapons are located only before or after the more challenging sections; wearable

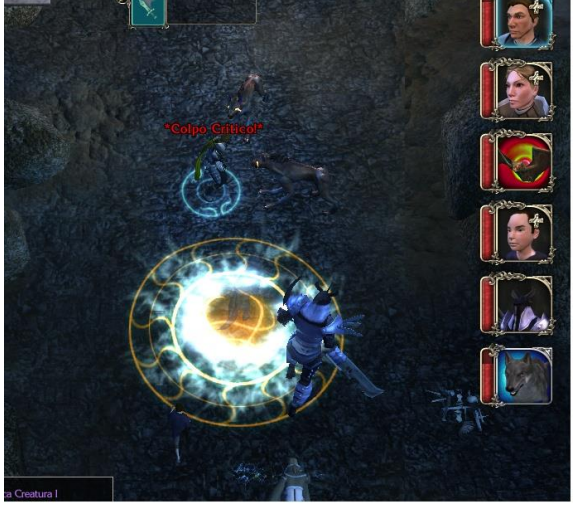

(a) The "group fight" task

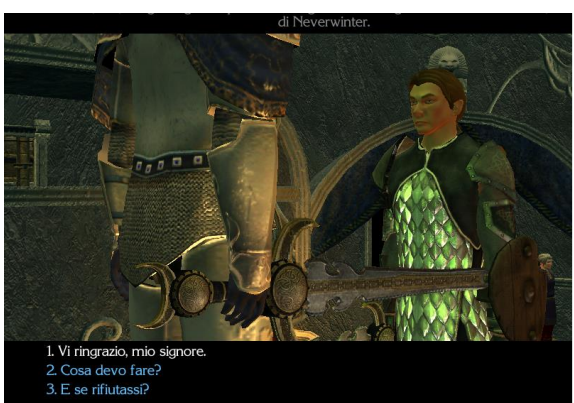

(b) The "animated dialogue" task

Fig. 7 Tasks in the Flow game level.

objects increase player's skills that can also grow using the experience points.

There is a party of skilled and useful allies (wizard, warrior, thief) with different ways to enlist them (automatic, dialogue, payment): they are interactive with a proactive AI and, moreover, additional non-interactive NPCs like guards and peasants can also help the player.

Dialogues are useful and articulated providing useful information like memories, opinions and narrative elements (Fig. 7-b) while some of them influence the prosecution with moral entanglements; they have animations and camera shots and three of them use professional voice-dubbing to stimulate involvement.

An opening dialogue introduces the story-plot, assigning a goal to the player. The area features two combats with an helping NPC and a riddle task. The following area presents voice-dubbed dialogues and an indoor castle setting; the background music has a fast pace and a wizard ally automatically joins the party. Area 3 provides an optional path, a group fight, and a dialogue with moral entanglements with the boss enemy. The next area is even more challenging and ends with a voice-dubbed moral dialogue about player's greed, where the wrong choice will lead to an impossible fight against a giant dragon. The last area is placed in the 


\begin{tabular}{|c|c|c|}
\hline a & b & \multirow{3}{*}{ (1 } \\
\hline Mon Mar 03 16:56:16 2014, & ||attack_the_enemy_area1_enemy2, & \\
\hline Mon Mar 03 16:56:20 2014, & death_of_enemy_area1_enemy2, & \\
\hline a & c & \multirow{4}{*}{$(2$} \\
\hline Mon Mar 03 16:56:16 2014, & area1_f, 184.064, 195.545, & \\
\hline \multicolumn{2}{|c|}{$0.958389,0.420899,0.302280,0.581455,0.339574$} & \\
\hline \multicolumn{2}{|r|}{ d } & \\
\hline a & c & \multirow{4}{*}{ (3) } \\
\hline Mon Mar 03 16:56:16 2014 & area1_f, 184.064, 195.545, & \\
\hline 117 & e & \\
\hline $\begin{array}{l}4140,4230.26,4508.72,3 \\
4707.18,4343.59,4455.9\end{array}$ & $\begin{array}{l}\text { 718.46, 3771.28, 4288.72, 4334.36, } \\
4005.13,4274.36,4316.92,4463.59\end{array}$ & \\
\hline
\end{tabular}

Fig. 8 Entries from the three data logs. (1) timestamp (box a) and its related event (box b); (2): timestamp, map coordinates (box c) and five affective values (box d); (3): timestamp, map coordinates and raw sensor values (box e).

King hall, and presents the positive ending and the player's reward.

\section{Tools and Technologies}

The Emotiv EPOC headset is a wireless neuro-signal acquisition and processing system with 14 wet sensors (+2 reference), capable of detecting brainwaves at 128 $\mathrm{Hz}$ sequential sampling rate; sensors are placed around the pre-frontal and frontal brain regions according to the international 10-20 standard system and this process is safe because the headset is a passive measuring device, less invasive than other physiological methods.

The choice of Neverwinter Nights 2 has been taken by considering:

- gameplay assets rich of quests, characters and events

- large community with support, tools and mods

- a visual editor (Electron) used even by authors to create dialogues, NPCs, AI, triggers, visual assets

- powerful C-like scripting language ( NWScript) that allows to manage internal game variables

- XML widgets to customize the game interface

For the experimentation there are two types of data to extract: internal (environmental and character variables, map coordinates) and external (coming from the headset). We used NWNX4 [80] to deploy a C++ plugin useful for the communication between Neverwinter Nights 2 and the EEG headset; the tool is opensource and allows to exchange textual messages using the server version of the game: in this way, a game script calls the plugin methods to iteratively process the internal and the external data.

\section{Experimental Study}

As seen in [81] laboratory experimentation originally involved 13 volunteers male academic students tested in a range hour between 10.00 AM and 07.00 PM on weekdays. For this work we performed further experiments: 6 volunteers female academic students were added to balance dataset plurality and avoid skewed results by having about $1 / 3$ of female population. The time duration of an experimental session was about one hour and half in which subjects were in a silent, empty and comfortable office room, seated on an adjustable chair.

The first Setup step consists in headset and sensors placing with signal tuning; after this, a brief explanation about modalities of the study was provided followed by the compilation of a pre-questionnaire about gaming preferences. The next step is the Tutorial that allows to familiarize with the user-interface: an experimenter helps the player to perform tasks like "move the character", "rotate and zoom the view", "talk with a NPC", "open the inventory or a chest and use items", "fight an enemy", "steal an object". We consider these steps a way to induce relaxation and a neutral initial affective mood (similar to $[28,38,82]$ ).

A gaming session starts by entering the first level and the NWNX plugin begins to record the EEG and game data; after the first game level a postquestionnaire about the player experience is compiled and the same happens after the second one. Each subject uses the same game character with all statistics set to average values. Different data sources are available for each session:

- a video capture of the gaming session

- a pre- and post- questionnaire

- three log files featuring the synchronized game and EEG data

Fig. 8 shows entries from the data files, each with common and specific fields. The events log (Fig. 8-1) contains the timestamp and the tag of the event; the affective log (Fig. 8-2) has the name of the area, the XY coordinates and the punctual values of the emotions, classified by the Emotiv EPOC internal algorithms (range between 0 and 1); the raw $\log$ (Fig. 8-3) collects the punctual values of the 14 headset sensors.

A game capture video shows a custom XML UIPane widget used by NWNX to print on the screen the same timestamp that synchronizes the data (Fig. 9); note that the data logs determine implicit annotations for these videos. 


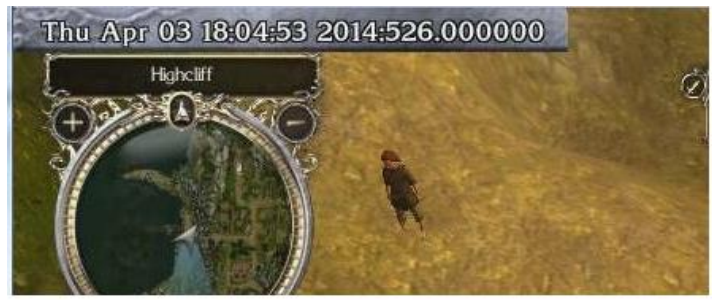

Fig. 9 the custom XML widget which prints the internal timestamp on the game screen.

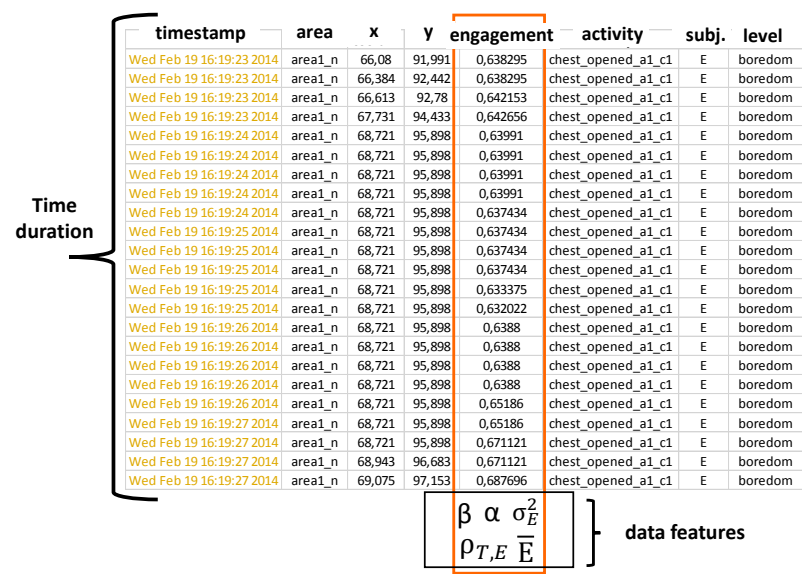

Fig. 10 A single activity in the affective log with the sequence of values that covers its time duration; five numerical features summarize the punctual values of "Engagement".

\section{Evaluation of EEG data}

A game level can be considered as the sequence of activities which a player faces during his gaming session: this approach allows an interpretation of data which greatly changes over time highlighting factors like repetitiveness, environmental changes and ludic interactions. For this study the activities considered are simple/dubbed/riddle dialogue, single/group fight, chest open, skills upgrade and stealing task.

We retrieved data by crossing the events log with the affective log: the first one provides the identifier and the initial time of the activity (Fig. 8-1) while the second supplies the affective values, synchronized by the same timestamp (Fig. 8-2). The amount of affective data in the entire time duration is given by the sequence of entries between the initial timestamp and one that precedes the next event (Fig. 10).

In the Boredom game level, the subjects performed on average $4.8,6.1$, and 3.7 activities for the tree areas, respectively. In the Flow game level, they performed on average $6.8,8.6,8.3$, and 3.2 , activities for the four areas, respectively. Only 3 subjects managed to reach the Flow level final area. Overall, more event types are available for the Flow level, offering a progressive story- plot with challenges, that require time to evolve in a natural way to the player's eyes.

To handle objective affective data we chose a Support Vector Machine (SVM), a machine learning technique that performs a supervised binary classification, to classify each activity into the two states of Boredom or Flow. To train the model we use the label associated to each activity, considering the game level from which it comes. In this way it is possible to investigate if the brain-recorded data are characteristic enough to differentiate (separate) among the two sets of guidelines; if this occurs for a substantial proportion of activities, then player's emotions have changed during the gaming sessions, that is what we wanted to achieve.

The SVM deals with a set of points (feature vectors) $x_{i}$ along with their categories $y_{i}$, for some dimension $d$, the $x_{i} \in R^{d}$ and the $y_{i}= \pm 1$; we are looking for the best separating hyperplane, defined by a possibly small set of support vectors.

Some binary classification problems do not have a simple hyperplane as a separating criterion: for those problems there is a variant that retains the simplicity of SVM by applying the kernel trick, to fit the maximummargin hyperplane in a transformed feature space: the resulting algorithm is formally similar, but every dot product is replaced by a non-linear kernel function.

In this way the transformation may be nonlinear and the transformed space high dimensional so, although the classifier is a hyperplane in the transformed feature space, it may be nonlinear in the original input space; a common kernel in this class is the Gaussian $R a$ dial Basis Function (RBF) $G(x, y)=e^{-\frac{1}{2 \sigma^{2}}(x-y)^{\prime}(x-y)}$, for some positive number $\sigma$.

\subsection{Data Features and Results}

To practically use SVM each emotion associated to a game activity must have numerical features: a feature is a single value which summarizes the related sequence of entries in the affective log recognized during the time duration (Fig. 10). The features calculated for each of the five recorded emotions are:

- the angular coefficient $\beta$ and the intercept $\alpha$ of a regression line, calculated considering the time $T$ as a positive variable which constantly increases on the $\mathrm{x}$-axis; it gives indications on the presence of a trend for the emotion $E$

$E_{i}=\alpha+\beta T_{i}+\mu_{i}, i: 1 . . n$

where $\mu_{i}$ is the statistical error 
Table 1 Binary classification results for the two game levels

\begin{tabular}{rccc}
\cline { 2 - 4 } & Boredom & Flow & Tot. \\
\hline \hline original events & 513 (ext.) & 513 & 1026 \\
\hline classified events & 542 & 484 & 1026 \\
\hline true positives & 489 & 460 & 949 \\
\hline false negatives & 24 & 53 & 77 \\
\hline \hline recall & $95 \%$ & $89 \%$ & \\
\hline f1-score & $93 \%$ & $92 \%$ & \\
\hline accuracy & & & $92 \%$ \\
\hline
\end{tabular}

- the Pearson product-moment correlation coefficient between time $T$ and the emotion $E$ : a value $r_{e}>0.7$ evidences a strong local correlation that can be direct (positive sign) or inverse (negative sign).

$\rho_{T, E}=\frac{\operatorname{cov}(T, E)}{\sigma_{T} \sigma_{E}},-1 \leq \rho_{T, E} \leq+1$.

In particular, we used the Pearson correlation coefficient $r_{e}$ calculated on the $n$ affective values contained in the time interval:

$$
\begin{gathered}
r_{e}=\frac{\sum_{i=1}^{n}\left(T_{i}-\bar{T}\right)\left(E_{i}-\bar{E}\right)}{\sqrt{\sum_{i=1}^{n}\left(T_{i}-\bar{T}\right)^{2}} \sqrt{\sum_{i=1}^{n}\left(E_{i}-\bar{E}\right)^{2}}}, \\
-1 \leq r_{e} \leq+1
\end{gathered}
$$

- the arithmetic mean $\bar{E}$ and the variance $\sigma_{E}^{2}$ that give quantitative information on the affective values of emotion $E$

Considering the 19 subjects (13 male and 6 female) we have a $790 \times 25$ predictors matrix $M$ where rows represent the labeled activities (277 Boredom and 513 Flow) and columns their relative 25 features (five for each emotion); since the dataset is unbalanced, we increased the Boredom cases by randomly sampling 236 examples from the original ones, and added them to the dataset again.

After the extension, $M$ becomes $1026 \times 25$ and it is used to train the SVM in a "leave-one-out" setting with the RBF kernel: the system is trained $n$ times iteratively using $n-1$ cases and each time the non-linear classifier is tested with the correspondent example left out.

The use of non-linear kernel functions is a major change from [81] that brought a marked performance improvement (accuracy from $70 \%$ linear to $92 \% \mathrm{RBF}$ ); results in Table 1 are very positive and confirm the goodness for design and development phases highlighting a strong polarization between the classes: all metrics are over $90 \%$ with accuracy and f1-score almost the same for both classes.
Table 2 Mood-congruent events by area: Boredom game level

\begin{tabular}{cccc} 
& total (ext.) & correct & rate \\
\hline \hline area1 & 176 & 169 & $96 \%$ \\
\hline area2 & 211 & 201 & $95.3 \%$ \\
\hline area3 & 126 & 119 & $94.4 \%$ \\
\hline \hline Tot. & 513 & 489 & $95.3 \%$ \\
\hline
\end{tabular}

Table 3 Mood-congruent events by area: Flow game level

\begin{tabular}{cccc}
\cline { 2 - 4 } & total & correct & rate \\
\hline \hline area1 & 130 & 111 & $85.4 \%$ \\
\hline area2 & 163 & 143 & $87.7 \%$ \\
\hline area3 & 157 & 145 & $92.4 \%$ \\
\hline area4 & 60 & 58 & $96.7 \%$ \\
\hline area5 & 3 & 3 & $100 \%$ \\
\hline \hline Tot. & 513 & 460 & $89.7 \%$ \\
\hline
\end{tabular}

Table 4 Mood-congruent events by type: Boredom game level

\begin{tabular}{rccc}
\cline { 2 - 4 } & total (ext.) & correct & rate \\
\hline dialogue & 224 & 217 & $96.9 \%$ \\
\hline single fight & 136 & 125 & $91.9 \%$ \\
\hline chest opened & 153 & 147 & $96.1 \%$ \\
\hline Tot. & 513 & 489 & $95.3 \%$ \\
\hline
\end{tabular}

\subsection{Classification for game level areas}

The classification process also considers to which areas the correctly classified activities belong: in this way it is possible to understand what the best-designed parts of a game level are. Results are in Table 2 and 3.

In all Boredom areas activities are well-classified up to $94 \%$ suggesting that they were constantly recognized as repetitive and tedious; moreover it is remarkable that while the first two areas are the same, the third introduces small variations however sufficient to reduce the perceived boredom.

For the Flow game level, accuracy rating vary but it is always over $85 \%$ : they begin from $85.4 \%$ for the first area and increase to $96.7 \%$ for the fourth one: this gradual progression corresponds to what theorized in Section 4 and 5, highlighting the goodness of a player experience characterized by a progressive involvement with high Engagement and Excitement. 
Table 5 Mood-congruent events by type: Flow game level

\begin{tabular}{rccr}
\cline { 2 - 4 } & total & correct & rate \\
\hline \hline dubbed dialogue & 46 & 46 & $100 \%$ \\
\hline riddle dialogue & 18 & 18 & $100 \%$ \\
\hline dialogue & 159 & 145 & $91.2 \%$ \\
\hline single fight & 153 & 130 & $85 \%$ \\
\hline fight vs. a group & 37 & 35 & $94.6 \%$ \\
\hline chest opened & 85 & 71 & $83.6 \%$ \\
\hline skills upgrade & 11 & 11 & $100 \%$ \\
\hline stealing action & 4 & 4 & $100 \%$ \\
\hline \hline Tot. & 513 & 460 & $89.7 \%$
\end{tabular}

\subsection{Classification for activity types}

Another useful analysis for the evaluation is to inquire what the best classified activity types are (results are in Table 4 and 5).

In the Boredom game level (textual) dialogue is classified best (96.9\%) followed by chest opened at $96.1 \%$. Classification rating is always over $90 \%$ evidencing a correct development phase and sound guidelines supported by the poorness and repetitiveness while dialogues are about $1 / 3$ more numerous than other types (224 vs. 153 and 136).

In the Flow game level too, dialogues are classified best, with dubbed and riddle at $100 \%$ and simple ones at $91.2 \%$. The chest opened task is the only under $85 \%$ and, compared with that in Boredom level (-12.5\%), denotes that repetitiveness is better than item variety.

The single fight has the same number of events of dialogue but it is classified only at $85 \%(-6.9 \%$ compared to the other game level); fight vs. a group has a much lower number of events (153 vs. 37) but better characterization $(94.6 \%)$ for the Flow game level.

Considering other activities specifically developed for the Flow gameplay, stealing action and skills upgrade are both at $100 \%$ but they have a low number of examples due to their optionality in the gaming session.

\section{Evaluation of Questionnaires}

From pre-questionnaires appears that $52.6 \%$ of subjects uses PCs for 2-5 hours daily and $36.8 \%$ for more than 6 hours while the time spent to play videogames is between 4-11 and 12-24 hours per week for this group.

Interest about videogames is strong for $84.2 \%$ of subjects and $78.9 \%$ of them has previously played an RPG one (offline or online).

Considering the gaming platforms, $68.4 \%$ prefers PC, $21 \%$ game consoles and 10.5\% mobile platforms;
Table 6 Post-questionnaires - Boredom game level

\begin{tabular}{rcccc} 
& None & A little & Enough & A lot \\
\hline \hline Satisfaction & 2 & 15 & - & 2 \\
\hline Allies satisf. & 10 & 8 & - & 1 \\
\hline Dialogues satisf. & 9 & 10 & - & 0 \\
\hline Boredom perc. & 0 & 7 & 10 & 2 \\
\hline Frustration perc. & 5 & 6 & 7 & 1 \\
\hline Involvement perc. & 3 & 11 & 5 & 0 \\
\hline
\end{tabular}

Table 7 Post-questionnaires - Flow game level

\begin{tabular}{rcccc}
\cline { 2 - 5 } & None & A little & Enough & A lot \\
\hline \hline Satisfaction & 0 & 9 & - & 10 \\
\hline Allies satisf. & 1 & 9 & - & 9 \\
\hline Dialogues satisf. & 0 & 8 & - & 11 \\
\hline Boredom perc. & 4 & 11 & 4 & 0 \\
\hline Frustration perc. & 3 & 14 & 2 & 0 \\
\hline Involvement perc. & 0 & 4 & 14 & 1 \\
\hline
\end{tabular}

about technical aspects, plot is important for $84.2 \%$, $52.6 \%$ prefers graphic, $31.6 \%$ dialogues and only $21 \%$ audio assets (multiple answers were allowed).

\subsection{Post-Questionnaires}

The post-questionnaires in Tables 6, 7 compiled from the 19 subjects were closed-answer questions with only one choice allowed.

About player's general Satisfaction, for Boredom game level $78.9 \%$ of subjects is polarized towards low values (a little) while for the Flow one $52.6 \%$ expresses a lot, $47.4 \%$ little and nobody chooses none. This results entirely reflect our expectations for the first level and are reasonable for the Flow one.

The proposed Allies are evaluated in Question 2: the $94.7 \%$ of subjects dislikes the one in Boredom game level while, for the second game level they are divided between positive and negative judgment (56.6\% versus $47.4 \%$ ) highlighting the need to reconsider this aspect in the development phase.

Question 3 evaluates Dialogue satisfaction: subjects dislike them for Boredom game level (47.4\% none and $52.6 \%$ little acceptance) while, for the Flow one is almost the opposite with $57.9 \%$ a lot and $42.1 \%$ a little.

By considering the perceived Boredom, in Question 4 subjects express enough $(52.6 \%)$ and a lot (10.5\%) for Boredom game level while for the other one $21 \%$ expresses none and $57.9 \%$ a little: this polarization confirms the goodness of the proposed guidelines. 
About the perceived Frustration, for Boredom game level subjects are almost divided between positive and negative judgment ( $57.9 \%$ versus $42.1 \%)$ while in the Flow one frustration results absent or very low for $89.5 \%$ of them: by considering also Question 4 results, this emphasizes a connection between perceived boredom and the frustration that comes when a gamer is forced to play something that dislikes.

Question 6 treats the Involvement perceived by players, for which the majority is polarized between negative values for Boredom game level $(73.7 \%)$ and positive for the Flow one (78.9\%): this results fully confirm the decisions taken.

\section{Conclusions and Future work}

In this study we presented the formalization of guidelines for RPG videogames and their development in a famous commercial one; moreover a novel evaluation methodology based on machine learning was proposed, presenting encouraging experimental results.

Goodness of the design phase is notable both for the game level structures and for the implementation of their activities (tasks and events). Dialogues, the fulcrum of RPG gameplay, proved the ability to manipulate player's emotions positively and negatively and also the progression of skills and challenges and the repetitiveness variation appears from the experimentation.

The analysis of the raw affective data will be useful in order to extract brainwave trends and compare them with the results provided by the internal algorithms used here; further studies will inquire about emotional differences between male and female gamers while it is possible a further analysis of the tasks grouped according their type (duels, dialogues) with the aim to detect trends of their aroused emotions.

Forms of Dynamic Difficulty Adjustment (DDA) mechanisms [83] can benefit from this work, in fact with real-time classification it could be possible to change an affective mood during the gaming session. The recorded game videos might be automatically analyzed to extract gameplay dynamics like strategies and behavior patterns as done by Moura et al. [84] in Dragon Age Origins while also the AI of allies and NPCs can dynamically take advantage of the proposed classification method becoming more reactive toward the player.

Finally, given the extreme variety of game genres, it can be useful to improve the guidelines in other videogames featuring different gameplay and interaction modalities like in Obbink et al. [82] and Milam and El Nasr [85] with the aim to propose a framework to study and categorize game dynamics and assets.

\section{References}

1. Gee, J.P.: Learning and games. The ecology of games: Connecting youth, games, and learning 3 (2008) 21-40

2. Craigh, L.: Game taxonomies: A high level framework for game analysis and design. Gamasutra (April 2003)

3. Gonzalo, F.: Ludologists love stories, too: notes from a debate that never took place. In: Proceedings of the 2003 DiGRA International Conference. (2014)

4. Csikszentmihalyi, M.: Does being human matter? on some interpretive problems of comparative ludology. Behavioral and Brain Sciences 5 (3 1982) 160-160

5. Bergervoet, E.J., Sluis, F., Dijk, E.M.A.G., Nijholt, A.: Bombs, fish, and coral reefs. The Visual Computer 29(2) (2012) 99-110

6. Kallergi, A., Verbeek, F.J.: Video games for collection exploration: Games for and out of data repositories. In: Proceedings of the 14th International Academic MindTrek Conference: Envisioning Future Media Environments. MindTrek '10, ACM (2010) 143-146

7. Ardito, C., Buono, P., Costabile, M., Lanzilotti, R., Pederson, T.: Mobile games to foster the learning of history at archaeological sites. In: Proceedings of the IEEE Symposium on Visual Languages and Human-Centric Computing, IEEE Computer Society (2007) 81-86

8. Carter, M., Downs, J., Nansen, B., Harrop, M., Gibbs, M.: Paradigms of games research in hci: A review of 10 years of research at chi. In: Proceedings of the First ACM SIGCHI Annual Symposium on Computer-human Interaction in Play. CHI PLAY '14, ACM (2014) 27-36

9. Bayliss, J.D., Bierre, K.: Game design and development students: Who are they? In: Proceedings of the 3rd International Conference on Game Development in Computer Science Education. GDCSE '08, ACM (2008) 6-10

10. McGill, M.: Critical skills for game developers: An analysis of skills sought by industry. In: Proceedings of the 2008 Conference on Future Play: Research, Play, Share. Future Play '08, ACM (2008) 89-96

11. Potanin, R.: Forces in play: The business and culture of videogame production. In: Proceedings of the 3rd International Conference on Fun and Games. Fun and Games '10, ACM (2010) 135-143

12. Scavarelli, A., Arya, A.: Cindr: A proposed framework for ethical systems in video games. In: Games Media Entertainment (GEM), 2014 IEEE. (Oct 2014) 1-5

13. Snowdon, J., Oikonomou, A.: Games as a new medium for social criticism. In: 16th International Conference on Computer Games, CGAMES 2011, Louisville, KY, USA, 27-30 July, 2011. (2011) 101-106

14. Tyni, H., Kultima, A., Mäyrä, F.: Dimensions of hybrid in playful products. In: Proceedings of International Conference on Making Sense of Converging Media. AcademicMindTrek '13, ACM (2013) 237:237-237:244

15. Aarnoutse, F., Peursum, L., Dalpiaz, F.: The evolution of advergames development: A study in the netherlands. In: Games Media Entertainment (GEM), 2014 IEEE. (Oct 2014) 1-8

16. Zagal, J.P., Ladd, A., Johnson, T.: Characterizing and understanding game reviews. In: Proceedings of the 4th International Conference on Foundations of Digital Games. FDG '09, ACM (2009) 215-222

17. Paavilainen, J.: Critical review on video game evaluation heuristics: Social games perspective. In: Proceedings of the International Academic Conference on the Future of Game Design and Technology. Futureplay '10, ACM (2010) 56-65

18. Korhonen, H., Paavilainen, J., Saarenpää, H.: Expert review method in game evaluations: Comparison of two 
playability heuristic sets. In: Proceedings of the 13th International MindTrek Conference: Everyday Life in the Ubiquitous Era. MindTrek '09, ACM (2009) 74-81

19. Mandryk, R.L., Inkpen, K.M.: Physiological indicators for the evaluation of co-located collaborative play. In: Proceedings of the 2004 ACM Conference on Computer Supported Cooperative Work. CSCW '04, ACM (2004) 102-111

20. Picard, R.W.: Affective computing. Technical Report 321, M.I.T Media Laboratory Perceptual Computing Section (1995)

21. Nacke, L.: Affective Ludology: Scientific Measurement of User Experience in Interactive Entertainment. PhD thesis, Blekinge Institute of Technology, Karlskrona, Sweden (2009) http://phd.acagamic.com, (ISBN) 978-91-7295-1693.

22. Noah, J.A., Tachibana, A., Bronner, S.: Multi-core processing within the frontal lobe. In: Proceedings of the 6th International Conference on Foundations of Digital Games. FDG '11, ACM (2011) 280-282

23. Groenegress, C., Spanlang, B., Slater, M.: The physiological mirror: a system for unconscious control of a virtual environment through physiological activity. The Visual Computer 26(6-8) (2010) 649-657

24. Rawn, R.W.A., Brodbeck, D.R.: Examining the relationship between game type, player disposition and aggression. In: Proceedings of the 2008 Conference on Future Play: Research, Play, Share. Future Play '08, ACM (2008) 208-211

25. Jacopin, E.: Game ai planning analytics: The case of three first-person shooters. In: AAAI Conference on Artificial Intelligence and Interactive Digital Entertainment. (2014)

26. Gilleade, K.M., Dix, A., Allanson, J.: Affective videogames and modes of affective gaming: assist me, challenge me, emote me. In: In The 2005 International Conference on Changing Views: Worlds in Play. (2005)

27. Grimes, D., Tan, D.S., Hudson, S.E., Shenoy, P., Rao, R.P.: Feasibility and pragmatics of classifying working memory load with an electroencephalograph. In: Proceedings of the SIGCHI Conference on Human Factors in Computing Systems, ACM (2008) 835-844

28. Vachiratamporn, V., Moriyama, K., Fukui, K., Numao, M.: An implementation of affective adaptation in survival horror games. In: 2014 IEEE Conference on Computational Intelligence and Games, CIG 2014, Dortmund, Germany, August 26-29, 2014. (2014) 1-8

29. Lotte, F.: Brain-computer interfaces for 3d games: Hype or hope? In: Proceedings of the 6th International Conference on Foundations of Digital Games. FDG '11, ACM (2011) $325-327$

30. Carofiglio, V., Abbattista, F.: A rough bci-based assessment of user's emotions for interface adaptation: Application to a 3d-virtual- environment exploration task. In: Proceedings of the First International Workshop on Intelligent User Interfaces: Artificial Intelligence meets Human Computer Interaction (AI*HCI 2013) A workshop of the XIII International Conference of the Italian Association for Artificial Intelligence (AI*IA 2013), Turin, Italy, December 4, 2013. (2013)

31. Coulton, P., Wylie, C.G., Bamford, W.: Brain interaction for mobile games. In: Proceedings of the 15th International Academic MindTrek Conference: Envisioning Future Media Environments. MindTrek '11, ACM (2011) 37-44

32. Burke, J.W., McNeill, M.D.J., Charles, D.K., Morrow, P.J., Crosbie, J.H., McDonough, S.M.: Optimising engagement for stroke rehabilitation using serious games. The Visual Computer 25(12) (October 2009) 1085-1099
33. Kang, K.K., Kim, J.A., Kim, D.: Development of a sensory gate-ball game system for the aged people. The Visual Computer 25(12) (2009) 1073-1083

34. Nacke, L., Stellmach, S., Lindley, C.: Electroencephalographic assessment of player experience: A pilot study in affective ludology. Simulation and Gaming 42(5) (2011) $632-655$

35. Nogueira, P., Rodrigues, R., Oliveira, E., Nacke, L.: Guided emotional state regulation: Understanding and shaping player's affective experiences in digital games. In: AAAI Conference on Artificial Intelligence and Interactive Digital Entertainment. (2013)

36. Vourvopoulos, A., Liarokapis, F.: Brain-controlled NXT robot: Tele-operating a robot through brain electrical activity. In: Third International Conference on Games and Virtual Worlds for Serious Applications (VS-GAMES), Athens, May 4-6, 2011, IEEE (2011) 140-143

37. Liarokapis, F., Vourvopoulos, A., Ene, A., Petridis, P.: Assessing brain-computer interfaces for controlling serious games. In: Games and Virtual Worlds for Serious Applications (VS-GAMES), 2013 5th International Conference on, IEEE (2013) 1-4

38. Schwarz, D., Subramanian, V., Zhuang, K., Adamczyk, C.: Educational neurogaming: Eeg-controlled videogames as interactive teaching tools for introductory neuroscience. In: AAAI Conference on Artificial Intelligence and Interactive Digital Entertainment. (2014)

39. Chumerin, N., Manyakov, N.V., Combaz, A., Robben, A., van Vliet, M., Van Hulle, M.M.: Steady state visual evoked potential based computer gaming - the maze. In: Intelligent Technologies for Interactive Entertainment. Springer (2012) 28-37

40. Cheung, G.K., Zimmermann, T., Nagappan, N.: The first hour experience: How the initial play can engage (or lose) new players. In: Proceedings of the First ACM SIGCHI Annual Symposium on Computer-human Interaction in Play. CHI PLAY '14, ACM (2014) 57-66

41. Myers, D., et al.: Computer games genres. Play \& Culture 3(4) (1990) 286-301

42. Machado, M.C., Fantini, E.P.C., Chaimowicz, L.: Player modeling: Towards a common taxonomy. In: Computer Games (CGAMES), 2011 16th International Conference on. (July 2011) 50-57

43. Lewis, C., Whitehead, J., Wardrip-Fruin, N.: What went wrong: A taxonomy of video game bugs. In: Proceedings of the Fifth International Conference on the Foundations of Digital Games. FDG '10, ACM (2010) 108-115

44. Pinelle, D., Wong, N., Stach, T.: Using genres to customize usability evaluations of video games. In: Proceedings of the 2008 Conference on Future Play: Research, Play, Share. Future Play '08, ACM (2008) 129-136

45. Berens, K., Howard, G.: The Rough Guide to Videogaming. Miniguides Series. Rough Guides (2002)

46. Carlà, F.: Space invaders: la vera storia dei videogames. Castelvecchi (1996)

47. Khaleghi, K., Lugmayr, A.: Video game market segmentation based on user behavior. In: Proceeding of the 16th International Academic MindTrek Conference. MindTrek '12, ACM (2012) 283-286

48. Langer, R., Hancock, M., Scott, S.D.: Suspenseful design: Engaging emotionally with complex applications through compelling narratives. In: Games Media Entertainment (GEM), 2014 IEEE. (Oct 2014) 1-8

49. Frasca, G.: Simulation versus narrative: Introduction to ludology. In: The Video Game Theory Reader. Routledge (2003) 221-236 
50. Calleja, G.: Narrative involvement in digital games. In: Conference proceedings from Foundations of Digital Games. Chania, Crete, Greece. (2013)

51. Zagalo, N., Torres, A.: Character emotion experience in virtual environments. The Visual Computer 24(11) (2008) 981-986

52. Tatum, W.O.: Ellen r. grass lecture: Extraordinary eeg. The Neurodiagnostic Journal 54(1) (2014) 3-21

53. Russell, J.A.: A circumplex model of affect. Journal of personality and social psychology 39(6) (1980) 1161

54. Posner, J., Russell, J.A., Peterson, B.S.: The circumplex model of affect: An integrative approach to affective neuroscience, cognitive development, and psychopathology. Development and psychopathology 17(03) (2005) 715-734

55. Andreassi, J.L.: Psychophysiology: Human behavior \& physiological response. Psychology Press (2000)

56. Craveirinha, R., Roque, L.: Looking for the heart of interactive media: Reflections on video games' emotional expression. In: Proceedings of the 3rd International Conference on Fun and Games. Fun and Games '10, ACM (2010) 8-17

57. Jennett, C., Cox, A.L., Cairns, P., Dhoparee, S., Epps, A., Tijs, T., Walton, A.: Measuring and defining the experience of immersion in games. Int. J. Hum.-Comput. Stud. 66(9) (September 2008) 641-661

58. Chalmers, A., Debattista, K., Ramic-Brkic, B.: Towards high-fidelity multi-sensory virtual environments. The Visual Computer 25(12) (October 2009) 1101-1108

59. Choo, A., May, A.: Virtual mindfulness meditation: Virtual reality and electroencephalography for health gamification. In: Games Media Entertainment (GEM), 2014 IEEE. (Oct 2014) $1-3$

60. Dollard, J., Miller, N.E., Doob, L.W., Mowrer, O.H., Sears, R.R.: Frustration and aggression. Yale University Press (1939)

61. Park, J.Y., Park, J.H.: A graph-based representation of game scenarios; methodology for minimizing anomalies in computer game. The Visual Computer 26(6-8) (2010) 595605

62. Vanhatupa, J.M.: Guidelines for personalizing the player experience in computer role-playing games. In: Proceedings of the 6th International Conference on Foundations of Digital Games. FDG '11, New York, NY, USA, ACM (2011) 46-52

63. Thong, L.P.: Situated learning with role-playing games to improve transfer of learning in tertiary education classrooms. In: Games and Virtual Worlds for Serious Applications (VS-GAMES), 2014 6th International Conference on, IEEE (2014) $1-5$

64. Horsfall, M., Oikonomou, A.: A study of how different game play aspects can affect the popularity of role-playing video games. In: CGAMES, IEEE Computer Society (2011) 6369

65. Tychsen, A., Hitchens, M., Brolund, T.: Motivations for play in computer role-playing games. In: Proceedings of the 2008 Conference on Future Play: Research, Play, Share. Future Play '08, ACM (2008) 57-64

66. Lankoski, P.: Models for story consistency and interestingness in single-player rpgs. In: Proceedings of International Conference on Making Sense of Converging Media. AcademicMindTrek '13, ACM (2013) 246:246-246:253

67. Brown, E., Cairns, P.: A grounded investigation of game immersion. In: CHI'04 extended abstracts on Human factors in computing systems, ACM (2004) 1297-1300

68. Calleja, G.: In-Game: From Immersion to Incorporation. The MIT Press (2011)

69. Wilcox-Netepczuk, D.: Immersion and realism in video games - the confused moniker of video game engrossment. In: CGAMES, IEEE Computer Society (2013) 92-95
70. Fisher, C.D.: Boredom at work: A neglected concept. Human Relations 46(3) (1993) 395-417

71. Csikszentmihalyi, M.: Beyond boredom and anxiety Jossey-Bass (2000)

72. Broin, D.O.: Using a criteria-based user model for facilitating flow in serious games. In: Games and Virtual Worlds for Serious Applications (VS-GAMES), 2011 Third International Conference on, IEEE (2011) 63-69

73. Warpefelt, H., Strååt, B.: Anti-heuristics for maintaining immersion through believable non-player characters. In: International Conference on the Foundations of Digital Games, Crete, Greece, May 14-17, 2013. (2013) 455-456

74. Burelli, P.: Virtual cinematography in games: Investigating the impact on player experience. In: International Conference on the Foundations of Digital Games, Crete, Greece, May 14-17, 2013. (2013) 134-141

75. Nakamura, J., Csikszentmihalyi, M.: The concept of flow. Handbook of positive psychology (2002) 89-105

76. Juul, J.: In search of lost time: On game goals and failure costs. In: Proceedings of the Fifth International Conference on the Foundations of Digital Games. FDG '10, ACM (2010) 86-91

77. Zagal, J.P., Altizer, R.: Examining rpg elements: Systems of character progression. In: Proceedings of the 2014 Conference on the Foundations of Digital Games. (2014)

78. James, B., Fletcher, B., Wearn, N.: Three corners of reward in computer games. In: Proceedings of the 8th International Conference on the Foundations of Digital Games. (2013)

79. Chen, J.: Flow in games (and everything else). Commun. ACM 50(4) (April 2007) 31-34

80. Stieger, I.: Neverwinter nights extender v.4. http://www. nwnx.org/

81. Balducci, F., Grana, C., Cucchiara, R.: Classification of affective data to evaluate the level design in a roleplaying videogame. In: Games and Virtual Worlds for Serious Applications (VS-Games), 2015 7th International Conference on, Skovde (September 2015) 1-8

82. Obbink, M., Gürkök, H., Bos, D.P.O., Hakvoort, G., Poel, M., Nijholt, A.: Social interaction in a cooperative braincomputer interface game. In: Intelligent Technologies for Interactive Entertainment. Springer (2012) 183-192

83. Baldwin, A., Johnson, D., Wyeth, P., Sweetser, P.: A framework of dynamic difficulty adjustment in competitive multiplayer video games. In: Games Innovation Conference (IGIC), 2013 IEEE International. (Sept 2013) 16-19

84. Moura, D., el Nasr, M.S., Shaw, C.D.: Visualizing and understanding players' behavior in video games: Discovering patterns and supporting aggregation and comparison. In: Proceedings of the 2011 ACM SIGGRAPH Symposium on Video Games. Sandbox '11, ACM (2011) 11-15

85. Milam, D., El Nasr, M.S.: Analysis of level design 'push \& pull' within 21 games. In: Proceedings of the Fifth International Conference on the Foundations of Digital Games. FDG '10, ACM (2010) 139-146 